\title{
XVI.
}

\section{Die in Halle aufgefundenen Leibnitz-Briefe}

(zweite Folge)

im Auszug mitgetheilt

von

Iudwig Stein in Zürich.

Die zweite Gruppe. der anfgefundenen Leibnitz-Briefe, die ein besonderes Convolut bildet, enthïlt, wie ich bereits angedeutet habe '), Originalbriefe des Leibnitz an verschiedene Gelehrte und Staatsmänner. Diese Sammlung besteht aus 13 Briefen (Nr. 89 bis 101), die sehr verschieden an Inhalt und Werth sind. Von diesen 13 Briefen ist nur ein einziger gedruckt (Brief 99 vom 24. März 1699 an Joh. Andr. Stisser, Professor in Helmstädt). Aber auch dieser Brief, der an einem ganz entlegenen, wenig beachteten Ort abgedruckt ist (in den Annales Academiae Juliae vom Sommersemester 1722, Brunsvici, 1723), ist fast ganz unbekannt.

ron den restlichen 12 Briefon sind fünf historischen Inhalts (Nr. 91, 92, 95, 96 und 97), drei privater Natur und ohne Belang (No. 89, 93 und 98); die übrigen, fünf endlich, die ich vollinhaltlich wiedergebe, bieten zum Theil biographisches, zum Theil philosophisches Material.

Die auf die Geschichte bezüglichen Briefe, an die Geschichtsforscher Bose in Jena und Meibom in Helmstädt gerichtet, haben zumeist Untersuchungen über den Stammbaum der Welfen zu ihrem Inhalt. Die in diesen Briefen behandelten historischen

') Vgl. Archiv für Gesch. d. Philos. Bd. I, IIeft 1, S. 80. 
Fingen sind jedoch durch die Reiso des I,oibnit\% nach Italien behufs genauer lirmittlung und Feststellung des welfischen Stammbaums durchweg hinfillig geworden. Denn dic überraschenden Fundo an unbokanntem Material, wolche Leibnitz in Modena gemacht hatte, warfon alle Vermuthungen Meiboms so schr über den Haufen, dass Loibnitz in dem hochinteressanten Brief, den ich am Schlusse dieses Aufsatzes veröffentliche, die alte Streitfrago über den Stanmbaum der Welfen für endgültig geschlichtet und beigolegt hält. Es will mich daher bedünken, dass diese Leibnitzischen Briefe historischen Inhalts selbst Geschichtsforschern nur geringes Interesse bieten werden.

Biographisch wichtig ist nun ein Brief L.'s an den Syndicus Anderson in Hamburg (Nr. 80 der Sammlung). Ist schon der Inhalt - eine Aneiferung zur Fortsetzung der Studien über die Teutonen - recht bemerkenswerth, so gewinnt der Brief besonders durch zwei Nebenmomente einen erhöhten Werth. Einmal ist er deshalb bedeutsam, weil ihn Leibnitz sechs Wochen vor seinem Tode geschrieben hat, andermal ist er darum werthvoll, weil er deutlich durchblicken lässt, dass L.'s Verhältniss zum Grafen Bernstorf im letzten Lebensjahr des Philosophen denn doch kein so gespanntes gewesen sein konnte, als gemeiniglich angenommen wird ${ }^{2}$ ). Um so unbegreiflicher und unverzeihlicher ist die Missachtung, die der hannöversche Hof beim Tode jenes Mannes bekundete, der die kostbarsten Jahre seines Lebens daran gewendet hatte, den Glanz eben dieses Hofes nach allen Richtungen hin zu verbreiten. Wäre Leibnitz wirklich in völlige Ungnade gefallen, wie man bisher annahm, so liesse sich die kalte Theilnahmslosigkeit des Hofes beim 'Tode des Philosophen wenigstens begreifen, wonn auch nicht entschuldigen. Aber der gleich anzuführende Brief an Anderson zeigt ja deutlich, dass von einem Abbrechen aller Verbindungen mit Leibnitz seitens des Hofes nicht eutfernt die Rede sein kann, da der Minister Bernstorf doch noch unmittelbar vor L.'s Tode ihm einen Auftrag ertheilt, also sich noch der Feder des Philosophen bedient hat, wohl um sich den Syndicus

2) Vgl. namentlich Guhrauer, Leibnitz, II, S. 324. 
von Hamburg durch einige verbindliche Worte zu Danke zu verpflichten. Und als Leibnitz sechs Wochen darauf starb, bewies der Hof eine so unzarte Lieblosigkeit, dass ein schottischer Edelmann, der dem Leichenbegängnisse des Philosophen beiwohnte, wohl in etwas übertreibender Weise, den Ausspruch that ${ }^{3}$ ): "er wurde eher wie ein Wegelagerer begraben, als wie ein Mann, der die Zierde seines Vaterlandes gewesen war".

Der Brief an den Syndicus Anderson lautet:

\section{Monsieur}

Quoyque je doute fort si cette lettre vous pourra encor trouver à Paris je prend la liberté de vous l'écrire, encouragé par Mons. de Bernstorf. il se souvient avec beancoup de plaisir de ce qu'il a remarqué de vos lumières sur l'ancien Teutonisme. il croit que vous aurés pî trouver l'occasion à Paris, de les augmenter; et il souhaite d'eu avoir quelques nouvelles. Nous sommes un peu ici dans votre goust, Monsieur, et nous encourageons et aidons Monsieur Eccard dans le louable dessein d'y travailler. Nous vous aurons tout de l'obligation de vos assistances, et au reste, vous souhaitant toute sorte de satisfaction dans votre voyages je suis avec zèle Monsieur

Hannovre le 25

de Sept. 1716. votre très humble et obeissant serviteur

Einen reichen Einblick in jene wunderbare, einzig dastehende Vielseitigkeit der wissenschaftlichen Probleme, an die Leibnitz seine geniale Geisteskraft gesetzt hat, gewährt uns sein Brief (Nr. 94) an den Professor H. v. d. Haardt in Helmstädt. Hier handelt er mit meisterlicher Klarheit im knappsten Ralımen über die heterogensten Gebiete, die mit einander nur sehr lose zusammenhïngen, wie über den Ursprung der Sitten, speciell einiger Gebräuche der Juden, über die Ssabier, über die Astronomie und Astrologie.

Der interessante Brief lautet:

Vir celeberrime, fautor Honoratissime.

Quas mihi inspiciendas miseras rerum judaicarum figuras sane pulcherrimas cum gratiarum actione decenti remitto, et magnopere

3) Vgl. Kuno Fischer, Leibnitz und seine Schule, S. 66. 
opto ut, quac de jure judaico moliris ritibusque antiquorum, praesertim quibus res patriarcharum illustrentur, reipublicae ne invideas. lloc profecto crit aperire rerum sacrarum fontes. Mihi quicquid licant quidam nostri mirifice Spenceri et similium institutum placet, qui causas juris divini positivi quaerunt in populorum ritibus, qui ipsi saepe fundamentum habent in natura rerum. Sane circumcisionem apud nonnulos populos necessitate adhiberi, ex itinerum scriptionibus didicimus. Sunt enim quibus praeputium nimis excrescit, et corneam duritiem acquirit. Ex tali tribu credibile est vel fuisse Abrahamum, vel cum ad tales populos itinera suscipere cogitaret, veritum, ne posteris suis ea labes veniret. Quod mihi perinde videtur ac si quis ad Russos et Tartaros cogitans cum suis, aut ex illis regionibus oriundus vel certe plicam Russicam, quae vulgo Polonica dicitur, metuens eam legem familiae suae diceret, ut maturi capilli resecarentur. Quod si idem propheta esset, et voluntatis divinae commercia praestaret mortalibus, suamque gentem peculiari ratione divino Ministerio mancipare institueret, posset ritum utilem hunc ferre in foederis autoramentum.

De Sabaeis vellem nostris plura essent nota, eorum Theologiam forte sine Maimonide et quibusdam Arabibus prorsus ignoraremus. Avide expecto Alcorani editionem patavinam ex ipsis Arabum commentariis illustratam. Nam Alcoranus continet antiquissimarum apud Arabes rerum non contemnenda vestigia. Astrologica antiqua a Sabaeis vel Zabiis (nam idem puto) fluxit. Ajunt eos, qui hodie Christiani S. Thomae dicuntur, plurima veterum institutorum retinuisse.

Rogo ut aliquando mihi per otium dicas Sententiam tuam, de aliquot Jobi locis, in quibus fit mentio rerum Astronomicarum. Spencerus et Marshamus utiliter usi sunt etiam Aegyptiorum rebus ad res judaicas illustrandas.

Putem ego etiam Astrologiam veterem (etsi nugalem) cujus tradita Vettius Valens et Ptolemaeus nobis pro parte servarunt, cum fere a Chaldaeis et Aegyptiis fluxerit, in rebus sacris illustrandis profu-

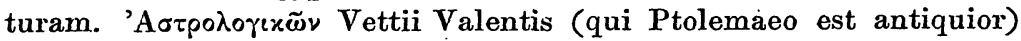
fragmentum edidit Joach. Camerarius. Integrum opus vidi apud 
summae doctrinae virum Petrum Danielem Huetium (P. Huet), episcopum, nunc Abrincensem in Normannia Gallorum. Aliud in Anglia extare accepi. Huetius ingenis de Editione cogitarat, quae noscendis Antiquorum Editionibus sane.utilis foret.

Opto ut consilia tua Smo. duci Rud. Aug. data de redimendis Mins. Hinkelmanni succedant.

Vale dabam Hann. obsequentissimus

10 April $1695 . \quad$ Godefridus Guilielmus Leibnitius.

P. S. Specimina quaedam meditationum tuarum circa patriarcharum jura aliquando intelligere iucundissimum erit. Pro ectypo bullae indulgentiariae gratias repeto.

Bei der vorsichtigen Art, die Leibnitz theologischen Dogmen gegenüber sich bewahrt hat, kann es wohl überraschen, wie er hier mit rückhaltsloser Offenheit den Ursprung der Beschneidung, entgegen dem Wortsinn der Bibel, auf eine altheidnische Sitte zurückführt. Dieses unbefangene Hinwegsetzen über die religiöse Tradition lïsst denn doch vermuthen, dass er über religiöse Dogmen weit rationalistischer gedacht hat, als Pichler zugeben möchte ${ }^{+}$).

Seine Werthschätzung des Maimonides als Quelle für die Ssabier zeugt von einem feinen historischen Verständniss. Die neuere Forschung über die Ssabier hat die Vermuthung unseres Philosophen glänzend bestätigt ${ }^{5}$ ). Wie denn Leibnitz überhaupt der erste unter den neueren Philosophen gewesen ist, der die Bedeutung des More Nebûkhim des Maimonides für die Geschichte der mittelalterlichen Philosophie erkannt und ausreichend gewürdigt hat $^{6}$ ).

Einen wichtigen Beitrag zur Characterisirung der naturwissenschaftlichen, speziell chemischen Kenntnisse des Leibnitz liefert folgender Brief (Nr. 100) an Prof. Joh. Andr. Stisser in Helmstädt:

*) V'gl. Pichler, die Theologie des Leibnitz, passim.

5) Vgl. Chwolson, die Ssabier.

6) Leibnitz hat sich bekanntlich Excerpte aus dem More Nebûkhim gemacht, die er sodann mit Glossen versehen hat, vgl. Foucher de Careil, Leibnitz, la philosophie juive et la Cabbale, Paris, 1861. 
Vir Nobilissime et releberrino Fautor honoratissime.

Ante anni decursum acto me alieno exolvere conor. Reperio autem 'luis allhuc responsum deberi.

Quicstio est magni momenti an vera salium detur transmutatio, quase larvae suspecta non sit; id enim memini a quibusdam in dubium vocari, et constat quam facile ista corpora et tegant so et redetegant, quod resuscitatio Nitri Glauberiana ostendit, quam deinde Boylius excoluit. Unde sunt qui suspicantur, in Spiritu. Nitri ipsum Nitrum tenuissimas tantum in partes fortissime agitatas dispersum latere idemque de spirita salis iudicant, elementis cujusque seu immutarum partium figuris semper salvis. Sed horum everteretur Hypothesis, si liceret ex nitro salem parare communi similem, vel contra. ita enim sequeretur vel elementa unius mutari in elementa alterius, vcl communia esse utriusque elementa, quae sola sui varia affictione nunc nitrum constituant, nunc communem salem; cui sententiae favere videtur, quod spiritus salis a nitro abstractus dat spiritum nitri, qui si vere argentum iam, non aurum aggreditur vel admittenda transmutatio est, vel dicendum salina corpora in spiritu salis latentia, commutato cum nitri corpusculis loco, in fundo remansisse, illis evolantibus. Itaque examinandum esset quale sit caput mortuum residuum, et an non salis communis habeat naturam, quo casu magis esset transplantatio quam transmutatio; quemadmodum spiritum nitri a sale communi abstrahendo manet in fundo nitrum, spiritus salis prodit; quae sane elegans decussatio est, fere qualis in cementatione Cinnabaris cum argento. quodsi ex uno sale alterius spiritus haberi posset, vel contra, altero non assumto nec reliquo, praeclusa contratendentibus effugia essent.

Memini aliquando heteroclitum salem mecum communicari, cuius figura a potestate dissidebat; rem habeo inter adversaria, sed nunc memoriae non satis succurrit. Salem ammoniacum nativum haberi testis sum oculatus, vereorque adeo ut vera sint, quae de compositione ex fuligine, sale communi et urina tanquam concursu trium regnorum memorantur, cum mihi pure mineralis videatur. Celeberrimum Bohnium alicubi nescio quod exemplum salis fundamentaliter transmutati produxisse narravit quidam, locum 
non indicavit, sed haec omnia tuo ingenio tuaque experientia magis poterunt illustrari.

Vale. Dabam Guelfebyti 12 Dec. 1699.

Deditissimus

G. G. Leibnitius.

Der letzte Brief (101) dieser zweiten Gruppe von Autogrammen hat keine Adresse. Aber aus dem Inhalt desselben kann man mit apodictischer Gewissheit schliessen, dass er an den hannöverschen Minister Graf Bernstorf gerichtet war. Schon der Titel Excellenz, den L. dem Adressaten beilegt, führt uns auf die richtige Spur. Weiss man nun vollends, dass Graf Bernstorf L. den Auftrag zu dieser Reise nach Italien gegeben hat, mit welcher der doppelte Zweck verbunden war, einerseits den Stammbaum des $\mathrm{W}^{\top}$ elfenhauses $\mathrm{zu}$ eruiren, andererseits eine Familienverbindung zwischen diesem und dem Hause Este anzubahnen ${ }^{7}$ ), so kann man selbst beim flüchtigen Durchlesen kaum noch zweifelhaft sein, dass Graf Bernstorf der Adressat sein muss. Zum Ueberfluss besitzen wir noch einen gedruckten Brief L.'s an Bernstorf ${ }^{8}$ ), in welchem er ihm ausdrücklich die Initiative und den Auftrag zur italienischen Reise fast mit denselben Ausdrücken zuschreibt, wie am Schlusse des nachfolgenden Briefes:

\section{Monsieur.}

Je ne doute point que V. Ex ${ }^{\text {ce }}$ 'ait receu mes lettres passées, ou j'ay rendu compte de temps en temps de mes voyages.

Dernierement j'ay esté quasi six semaines à Modene, ou S. A. S. m'a fait communiquer quantité de Manuscrits. J'ay trouvé que les Historiens d'Este ont manqué entierement aux choses essentielles, et qu'ils n'ont pas connu la véritable connexion des deux Serenissimes Maisons, ayant mal rapporté et les temps, et les noms. d'ou il est arrivé, que des trés habils hommes en France ont douté de la connexion des maisons de Bronsvic et d'Este,

7) Vgl. Guhrauer, Leibnitz II, $87 \mathrm{ff}$.

8) Dieser Brief, datirt aus Wien vom 6./16. Mai 1698, ist abgedruckt bei Feder, Lettres choisies de la correspondance de Leibnitz, IIannovire 1805, p. $206-208$. 
nussi bien que Messicurs Meibom (vgl. eben Jrief 96 und 97) et Sagittarius qui m'ont communiqué leur doutes. Maintenant j'ay tout découvert; ayant trouvé les lieux de la demeure du progenitcur commun, et les lieux de la sepulture des anciens Marquis; ot même l'Epitapho de la fameuse Cunigonde héretiere des Guelfes, épouse du Marquis Azo, père de Guelfe, duc de Bavière et tige des Ducs de Bronsvic, avec une inscription admirable, dont le commencement est:

dicta Guniguldis regali Stemmate fulsi

indole nobilior nullus in orbe fuit

Germine Welfontis magni sum nata Alemanni.

Elle a esté enterrée dans une Abbaye, appartenante à présent aux Vénitiens, et dont le Cardinal Ottoboni, a present pape, a esté l'Abbé commendataire. A Modene même on ne scavoit rien de ces choses, mais un Religieux à Pise, curieux des antiquités, qui avoit esté autres fois dans cette Abbatie, m'en avoit donné quelques indices, qui m'ont fait deterrer le reste, quand je suis venu à Modène. il $y$ a encor une Abbaye dans le voisinage, ou j'ay appris, que les anciens Marquis dont ceux d'Este sont descendus, ont fait des fondations. J'y iray au premier jour, attendant les adresses d'un amy. Apres cela je me hasteray pour retourner en Allemagne, et pour ranger mes memoires, bien differens, de ce que j'aurois pû donner, si je n'avois eu ces conjonctures. J'ay mêmes trouvé une chose que je n'aurois jamais crû, c'est que Henry le Lion a eu le dominium directum du chasteau d'Este, l'ayant concedé, ou plustost confirmé en fief aux Marquis d'Este ses parens de la branche italienne. Et je puis expliquer commes toutes ces choses sont allées, et j'ay les extraits des investitures.

Enfin je crois de pouvoir estre satisfait de mes peines, qui n'ont pas esté petites, car pendant les six semaines que j'ay esté à Modène, je n'ay fait presque autre chose, que feuilleter: dans les vieux Manuscrits. Et il faut feuilleter long temps pour trouver quelque chose de bon. Aussi n'ay je pas en egard n'y à ma santé, qui n'est pas des. plus affermies, n'y à mes aises; pour satisfaire abondamment à ce que je croyays estre de mon devoir au prejudice de ma curiosité, qui pouvoit estre mieux contentée au 
couronnement d'Augsburg ou au moins un Carneval de Venise. Mais ces sortes de choses me tentent fort peu, lors qui'l s'agit de satisfaire à ce qu'on attend de moy. peut estre auroit on trouvé de plus heureux (au moins, pour se faire valoir) mais non pas facilement des plus appliqués. On enverra les effects dans les memoires que je vay rapporter.

J'espere aussi de jouir un jour des fruits de mes travaux, si dieu me donne assez de vie pour cela. Et c'est particulièrement sur vostre protection, Monsieur, que je fonde une bonne partie de ces esperances. Vous aves poussé ce dessein, vous en connoisses la consequence et j'espere que vous n'auries point lieu de vous en repentir. Je prie dieu de vous conserver long temps en bonne santé et je suis avec respect

Monsieur de vostre Excellence le tres humble et tres obeissant serviteur

Leibniz.

Venise $\frac{17}{17}$ de Fevrier 1690.

Wenn nun auch dieser Brief bisher völlig unbekannt war ${ }^{9}$ ), so erfährt doch unsere Kenntniss der Wirksamkeit L.'s in Italien durch denselben keine sonderliche Bereicherung. Deun über seinen genealogisch so wichtigen Fund in Modena sind wir bereits genügend unterrichtet, zumal I.eibnitz desselben häufig in seinen Correspondenzen erwähnt. Was uns in diesem Briefe besonders angenehm anmuthet, ist der fast jugendlich frische Ton und die zuversichtliche Art, mit welcher er über. den vermeintlich so hoch wichtigen Fund berichtet. Man merkt dem Briefe die fröhliche Stimmung des Entdeckers, der den längstvermutheten Schatz nach unsäglichen Mühen endlich gehoben hat, fast in jeder Zeile an. Recht bezeichnend ist auch die Schlusswendung des Briefes, in welcher ILeibnitz auf den wohlverdienten Dank des hannöverschen

9) Herr Geh. Rath C. J. Gerhardt in Eisleben, der ausgezeichnete Kenner und Herausgeber der philosophischen Schriften des Leibuitz, hat mir auf meine Anfrage mit dankenswerther Liebenswürdigkeit bestätigt, class vorstelıender Prief des Philosophen an Bernstorf noch nirgends gedruckt ist. 
Ilauses anspielt, der ihm hoffentlich nicht ausbleiben werde. Wie traurig sich unser Plilosoph aber gerade darin verrechnet hat, habe ich obch beroits angedeutet.

In cinem nächsten Aufsatz, der einige bisher ungedruckte Briefe L.'s ïber das Wesen und den Werth der Geschichte der Philosophie enthalten wird, gedenke ich die Yeröffentlichungen aus den Hallenser Leibnitz-Briefen zu beschliessen. 